A. Bohm A. Mostafazadeh

H. Koizumi Q. Niu J. Zwanziger

\title{
The Geometric Phase \\ in Quantum Systems
}

Foundations, Mathematical Concepts, and Applications in Molecular and Condensed Matter Physics

With 56 Figures 


\section{Table of Contents}

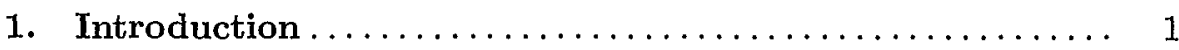

2. Quantal Phase Factors for Adiabatic Changes ......... 5

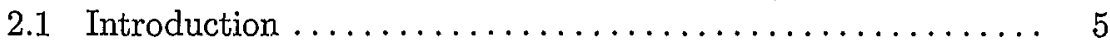

2.2 Adiabatic Approximation...................... 10

2.3 Berry's Adiabatic Phase ...................... 14

2.4 Topological Phases and the Aharonov-Bohm Effect ....... 22

Problems ................................ 29

3. Spinning Quantum System in an External Magnetic Field 31

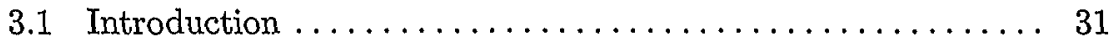

3.2 The Parameterization of the Basis Vectors ............ 31

3.3 Mead-Berry Connection and Berry Phase

for Adiabatic Evolutions - Magnetic Monopole Potentials.... 36

3.4 The Exact Solution of the Schrödinger Equation......... 42

3.5 Dynamical and Geometrical Phase Factors

for Non-Adiabatic Evolution .................. 48

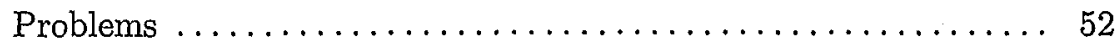

4. Quantal Phases for General Cyclic Evolution .......... 53

4.1 Introduction .............................. 53

4.2 Aharonov-Anandan Phase .................... 53

4.3 Exact Cyclic Evolution for Periodic Hamiltonians......... 60

Problems ................................... 64

5. Fiber Bundles and Gauge Theories $\ldots \ldots \ldots \ldots \ldots \ldots \ldots, 65$

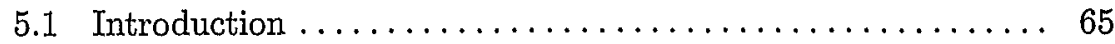

5.2 From Quantal Phases to Fiber Bundles.............. 65

5.3 An Elementary Introduction to Fiber Bundles.......... 67

5.4 Geometry of Principal Bundles and the Concept of Holonomy 76

5.5 Gauge Theories .......................... 87

5.6 Mathematical Foundations of Gauge Theories and Geometry of Vector Bundles ........................... 95

Problems ................................... 102 
6. Mathematical Structure of the Geometric Phase I:

The Abelian Phase . . . . . . . . . . . . . . . . . . . . 107

6.1 Introduction ............................ 107

6.2 Holonomy Interpretations of the Geometric Phase ........ 107

6.3 Classification of $U(1)$ Principal Bundles and the Relation Between the Berry-Simon and Aharonov-Anandan

Interpretations of the Adiabatic Phase .............. 113

6.4 Holonomy Interpretation of the Non-Adiabatic Phase

Using a Bundle over the Parameter Space ............ 118

6.5 Spinning Quantum System and Topological Aspects of the Geometric Phase ......................... 123

Problems .............................. 126

7. Mathematical Structure of the Geometric Phase II:

The Non-Abelian Phase . . . . . . . . . . . . . . . . . . . . 129

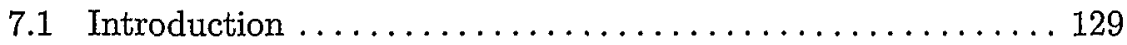

7.2 The Non-Abelian Adiabatic Phase . . . . . . . . . . . . . . . 129

7.3 The Non-Abelian Geometric Phase ................ 136

7.4 Holonomy Interpretations of the Non-Abelian Phase ....... 139

7.5 Classification of $U(\mathcal{N})$ Principal Bundles and the Relation Between the Berry-Simon and Aharonov-Anandan Interpretations of Non-Abelian Phase . ............. 141

Problems ....................................... 145

8. A Quantum Physical System in a Quantum Environment The Gauge Theory of Molecular Physics ............. 147

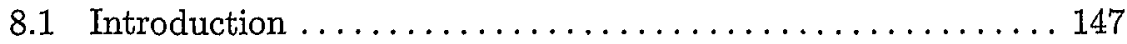

8.2 The Hamiltonian of Molecular Systems ............... 148

8.3 The Born-Oppenheimer Method ................ 157

8.4 The Gauge Theory of Molecular Physics............. 166

8.5 The Electronic States of Diatomic Molecule ............ 174

8.6 The Monopole of the Diatomic Molecule.............. 176

Problems ................................... 191

9. Crossing of Potential Energy Surfaces and the Molecular Aharonov-Bohm Effect........... 195

9.1 Introduction . . . . . . . . . . . . . . . . . . . . 195

9.2 Crossing of Potential Energy Surfaces ............... 196

9.3 Conical Intersections and Sign-Change of Wave Functions . . 198

9.4 Conical Intersections in Jahn--Teller Systems . . . . . . . . . . 209

9.5 Symmetry of the Ground State in Jahn-Teller Systems . . . . . 213

9.6 Geometric Phase in Two Kramers Doublet Systems . . . . . . . 219

9.7 Adiabatic-Diabatic Transformation ................. 222 
10. Experimental Detection of Geometric Phases I:

Quantum Systems in Classical Environments .......... 225

10.1 Introduction ............................... 225

10.2 The Spin Berry Phase Controlled by Magnetic Fields ..... 225

10.2.1 Spins in Magnetic Fields: The Laboratory Frame .... . 225

10.2.2 Spins in Magnetic Fields: The Rotating Frame ...... 231

10.2.3 Adiabatic Reorientation in Zero Field ............ 237

10.3 Observation of the Aharonov-Anandan Phase

Through the Cyclic Evolution of Quantum States........ 248

Problems .................................... 252

11. Experimental Detection of Geometric Phases II:

Quantum Systems in Quantum Environments......... 255

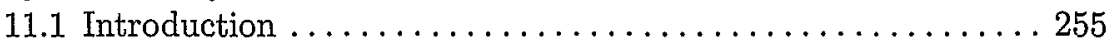

11.2 Internal Rotors Coupled to External Rotors............. 256

11.3 Electronic-Rotational Coupling ................... 259

11.4 Vibronic Problems in Jahn-Teller Systems ............ 260

11.4.1 Transition Metal Ions in Crystals .............. 261

11.4.2 Hydrocarbon Radicals ...................... 264

11.4.3 Alkali Metal Trimers...................... 265

11.5 The Geometric Phase in Chemical Reactions............ 270

12. Geometric Phase in Condensed Matter I: Bloch Bands ... 277

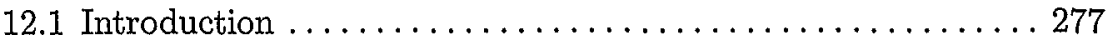

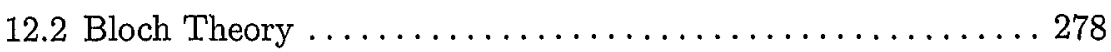

12.2.1 One-Dimensional Case . . . . . . . . . . . . . . . . 278

12.2.2 Three-Dimensional Case...................... 280

12.2.3 Band Structure Calculation ................. 281

12.3 Semiclassical Dynamics $\ldots \ldots \ldots \ldots \ldots \ldots \ldots \ldots \ldots . \ldots . \ldots 283$

12.3.1 Equations of Motion ....................... 283

12.3.2 Symmetry Analysis ........................ 285

12.3.3 Derivation of the Semiclassical Formulas ......... 286

12.3.4 Time-Dependent Bands ..................... 287

12.4 Applications of Semiclassical Dynamics............... 288

12.4.1 Uniform DC Electric Field. ................... 288

12.4.2 Uniform and Constant Magnetic Field ........... 289

12.4.3 Perpendicular Electric and Magnetic Fields ......... 290

12.4.4 Transport ............................... 290

12.5 Wannier Functions............................. 292

12.5.1 General Properties ....................... 292

12.5.2 Localization Properties........................ 293

12.6 Some Issues on Band Insulators ................... 295

12.6.1 Quantized Adiabatic Particle Transport .......... 295

12.6.2 Polarization ............................. 297

Problems .................................. 299 


\section{Geometric Phase in Condensed Matter II:}

The Quantum Hall Effect ......................... 301

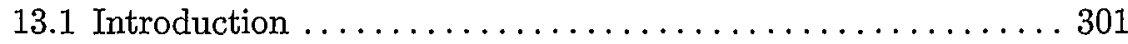

13.2 Basics of the Quantum Hall Effect .................. 302

13.2.1 The Hall Effect ....................... 302

13.2.2 The Quantum Hall Effect $\ldots \ldots \ldots \ldots \ldots \ldots . \ldots . \ldots 302$

13.2.3 The Ideal Model ........................ 304

13.2.4 Corrections to Quantization ................ 305

13.3 Magnetic Bands in Periodic Potentials .............. 307

13.3.1 Single-Band Approximation in a Weak Magnetic Field 307

13.3.2 Harper's Equation and Hofstadter's Butterfly . . . . . . 309

13.3.3 Magnetic Translations ..................... 311

13.3.4 Quantized Hall Conductivity ................ 314

13.3.5 Evaluation of the Chern Number ............. 316

13.3.6 Semiclassical Dynamics and Quantization ......... 318

13.3.7 Structure of Magnetic Bands and Hyperorbit Levels .. 321

13.3.8 Hierarchical Structure of the Butterfly .......... 325

13.3.9 Quantization of Hyperorbits

and Rule of Band Splitting ................ 327

13.4 Quantization of Hall Conductance in Disordered Systems . . . 329

13.4.1 Spectrum and Wave Functions ............... 329

13.4.2 Perturbation and Scattering Theory ............ 331

13.4.3 Laughlin's Gauge Argument .................. 332

13.4.4 Hall Conductance as a Topological Invariant ........ 333

14. Geometric Phase in Condensed Matter III:

Many-Body Systems ......................... 337

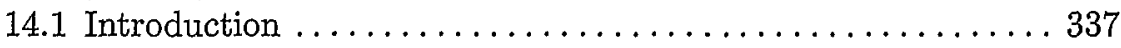

14.2 Fractional Quantum Hall Systems $\ldots \ldots \ldots \ldots \ldots \ldots \ldots \ldots . \ldots \ldots 33$

14.2.1 Laughlin Wave Function . ................ 337

14.2.2 Fractional Charged Excitations............... 340

14.2.3 Fractional Statistics .................... 341

14.2.4 Degeneracy and Fractional Quantization .......... 344

14.3 Spin-Wave Dynamics in Itinerant Magnets . . . . . . . . . . 346

14.3.1 General Formulation ..................... 346

14.3.2 Tight-Binding Limit and Beyond .............. 348

14.3.3 Spin Wave Spectrum...................... 350

14.4 Geometric Phase in Doubly-Degenerate Electronic Bands . . . . 353

Problem .................................... 359

A. An Elementary Introduction to Manifolds and Lie Groups 361

A.1 Introduction .............................. 361

A.2 Differentiable Manifolds ........................ 371

A.3 Lie Groups $\ldots \ldots \ldots \ldots \ldots \ldots \ldots \ldots \ldots \ldots \ldots \ldots \ldots \ldots \ldots$ 
B. A Brief Review of Point Groups of Molecules with Application to Jahn-Teller Systems ............ 407

References.............................. 429

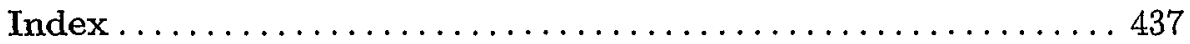

